\title{
The Effect of Delayed Chemotherapy on the Decrease of CAI25 in Epithelial Ovarian Cancer During Coronavirus Disease Pandemic in 2020
}

This article was published in the following Dove Press journal: Cancer Management and Research

\section{Qiang Yi \\ Yu Ran \\ Cong $\mathrm{Li}$ (D)}

Department of Gynecology, The First Affiliated Hospital of Chongqing Medical University, Chongqing, People's Republic of China
Correspondence: Cong Li

No. I YouYi Road, YuZhong District,

Chongqing 4000I0, People's Republic of

China

Tel +8615334506105

Fax+8623890II080

Email b05I04@I26.com
Objective: To investigate whether patients with epithelial ovarian cancer were affected by delayed chemotherapy during the coronavirus disease pandemic in 2020.

Materials and Methods: A delay of more than 21 days in the planned chemotherapy was defined as "delayed chemotherapy." Forty-five patients with epithelial ovarian, fallopian tube, and peritoneal cancer were delayed between January 1 and March 30, 2020 in the First Affiliated Hospital of Chongqing Medical University. Thirty-two cases were enrolled in this study. Neoadjuvant chemotherapy was used in 8 cases; palliative chemotherapy was used in 5 cases; and maintenance chemotherapy was used in 19 cases. Data included age, pathological type, surgical pathological stage, chemotherapy time and CA125 levels were collected. The half-life of CA125 and the decrease in CA125 levels before and after delayed chemotherapy were calculated.

Results: No patient got coronavirus disease. Compared with patients of ovarian cancer, fallopian tube epithelial cancer and peritoneal epithelial cancer in the same periods in 2019 , the half-life of CA125 in neoadjuvant chemotherapy group and recurrence chemotherapy group were more than 20 days, but there was no significant difference. Only when the delayed chemotherapy took place before CA125 turned negative, accompanied by an interval of more than 60 days, the CA125 half-life and the decreased range of CA125 were totally affected.

Conclusion: There was no evidence to support that once chemotherapy was delayed it would influence the decrease of CA125, but whether it would affect the long-term effects such as recurrence and five-year survival rate remains to be further followed up.

Keywords: epithelial ovarian cancer, CA125, delayed chemotherapy, half-life

\section{Introduction}

The coronavirus disease in 2020 has shown a global outbreak and become a worldwide healthcare concern. This disease can be asymptomatic, but can also devolve into acute respiratory distress syndrome in some cases. Patients with comorbidities are susceptible to aggressive infections and even death. The fatality rate for infected cancer patients in China is $28.6 \%$, compared to the rate of $2.3 \%$ observed in the entire population of coronavirus disease pandemic in $2020{ }^{1}$

Cancer patients need continuous care; for example, ovarian cancer patients need regular chemotherapy after diagnosis or operation, and their potential exposure to coronavirus disease pandemic in 2020 could carry a high risk or even become fatal. Therefore, on January 24, 2020, the Chinese government activated a first-level public 
health emergency response, for example, most hospitals stopped regular patient treatments, including those for cancer patients. On March 12, 2020, the government initiated a second-level response, and hospitals began to accept cancer patients cautiously and safely. Whether the delay in planned chemotherapy during this 2-month period showed adverse effects on cancer patients is unknown, and the extent of these effects remains to be elucidated. In this study, we examined the effects of delayed chemotherapy and calculated the decline in CA125 levels in epithelial ovarian cancer patients.

\section{Materials and Methods Data Collection}

From March 1 to June 30, 2020, 45 patients with ovarian epithelial cancer, fallopian tube epithelial cancer and peritoneal epithelial cancer were given delayed chemotherapy in the First Affiliated Hospital of Chongqing Medical University. They were treated with Paclitaxel combined with platinum chemotherapy, and chemotherapy types included maintenance chemotherapy after operation (6 times), palliative chemotherapy after recurrence and neoadjuvant chemotherapy (3-4 times). The study was approved by the appropriate Institutional Review Board of First Affiliated Hospital of Chongqing Medical University, and written informed consent was obtained from all subjects.

Exclusion criteria were as following: a) delayed chemotherapy due to adverse reactions of chemotherapy or other physical discomfort; b) with a normal CA125 level in the treatment of ovarian cancer, fallopian tube cancer and peritoneal epithelial cancer; c) those who change the plan in the course of chemotherapy: patients diagnosed as relapse after March 2020; d) those who are not satisfied with cytoreductive surgery (not reaching R0, R1); and e) incomplete data were collected.

Thirty-two patients were included. Data was collected from the beginning of treatment to the last chemotherapy including age, pathological type, surgical pathological stage, chemotherapy time and CA125. The interval of delayed chemotherapy was defined as more than 21 days ( 3 weeks).

In the same way, 49 cases with diagnosis as ovarian cancer, fallopian tube cancer and peritoneal epithelial cancer cases were collected in January 1 to March 30, 2019, and 32 cases were enrolled as control group.

\section{CAI25 Calculation}

CA125 half-life calculation method: formula: T1/2=DT/2log $(\mathrm{Ca} 1) /(\mathrm{Ca} 2)$, the half-life of CA125 is obtained. $\mathrm{Ca} 1$ is the concentration of CA125 in serum before treatment, and $\mathrm{Ca} 2$ is the value of CA125 in normal serum for the first time after operation. If the value of CA125 is high after operation, the lowest value in 3 months after operation should be used instead. DT is the number of days between $\mathrm{Ca} 1$ and $\mathrm{Ca} 2$.

The decrease of CA125: calculating the extent of CA125 level before and after delayed chemotherapy. The ratio of delayed chemotherapy before was calculated as $\left(\mathrm{Ca}^{-1}-\mathrm{Ca}\right) / \mathrm{Ca}^{-1}$, delayed chemotherapy after was calculated as $\left(\mathrm{Ca}_{-} \mathrm{Ca}^{+1}\right) / \mathrm{Ca}$. $\mathrm{Ca}^{-1}$ is the concentration of CA125 in serum before delayed chemotherapy happened, $\mathrm{Ca}$ is the concentration of CA125 in serum as delayed chemotherapy happened, $\mathrm{Ca}^{+1}$ is the concentration of CA125 in serum after delayed chemotherapy happened. "+" means decrease and "-"means increase.

\section{Statistical Methods}

All statistical analysis was conducted with the statistical computing and graphic drawing language. SPSS 23 statistical software was used for analysis. $T$-test was used for normal distribution data and nonparametric test was used for nonnormal distribution data, $\mathrm{P}<0.05$ was statistically significant. Sample less than 3 was not used for statistical analysis.

\section{Result \\ The Characteristic of Patients and the Comparison of CAI 25 Half-Life (TI/2) in 2019 and 2020}

Thirty-two cases in 2020 were analyzed as delayed chemotherapy. The ages were equal to 56-57 years old, the total $\mathrm{T} 1 / 2$ was not different $(\mathrm{P}=0.904)$ compared with 32 cases involved in 2019. Data was analyzed in sub-group, CA125 half-life was higher than 20 days in neoadjuvant chemotherapy group (23.05 \pm 5.03 VS $19.07 \pm 6.65)$ and palliative chemotherapy group (27.90 \pm 8.89 VS $19.17 \pm 6.72)$, but there was no significant difference compared with those in 2019. At the meantime, there were no difference in FIGO stage and Histologic type (see Table 1).

\section{Comparison of $\mathrm{TI} / 2$ and Decrease Extend in Sub-Group of Delayed Chemotherapy}

Six characters of delayed chemotherapy were divided as following: 1) the aim of chemotherapy, maintain chemotherapy or not; 2) FIGO stage, early or not; 3) histologic type, high grade serous nor not; 4) delayed chemotherapy 
Table I The Characteristic of Patients and the Comparison of CAI25 Half-Life (TI/2) in 2019 and 2020

\begin{tabular}{|c|c|c|c|c|c|}
\hline Characteristic & 2020 & TI/2 in 2020 & 2019 & $T I / 2$ in 2019 & $\mathbf{P}$ \\
\hline Age(y) & $56.03(36-69)$ & $18.08 \pm 8.35$ & $57.06(42-74)$ & $17.95 \pm 8.68$ & 0.904 \\
\hline \multicolumn{6}{|l|}{ Chemotherapy type* } \\
\hline NACT & $8(25 \%)$ & $23.05 \pm 5.03$ & 7 (2I.88\%) & $19.07 \pm 6.65$ & 0.21 \\
\hline PCT & $4(12.5 \%)$ & $27.90 \pm 8.89$ & 7 (21.88\%) & $19.17 \pm 6.72$ & 0.09 \\
\hline MCT & $20(62.5 \%)$ & $14.13 \pm 6.69$ & $18(56.25 \%)$ & $17.04 \pm 10.19$ & 0.30 \\
\hline \multicolumn{6}{|l|}{ FIGO stage } \\
\hline I & I (3.13\%) & 21.9289 & $3(9.38 \%)$ & 14.11 & I \\
\hline II & 10 (31.25\%) & $14.52 \pm 5.69$ & $4(12.5 \%)$ & $23.86 \pm 12.37$ & 0.69 \\
\hline III & 18 (56.25\%) & $18.63 \pm 8.08$ & 21 (65.63\%) & $16.85 \pm 6.65$ & 0.45 \\
\hline IV & $3(9.38 \%)$ & $25.40 \pm 15.06$ & $4(12.5 \%)$ & $20.7 I \pm 15.47$ & 0.71 \\
\hline \multicolumn{6}{|l|}{ Histologic type\& } \\
\hline High grade serous & $24(75 \%)$ & $18.35 \pm 9.34$ & 23 (71.88\%) & $17.22 \pm 8.23$ & 0.66 \\
\hline Endometrioid & I (3.13\%) & 16.505 & 4 (I2.5\%) & 17.88 & l \\
\hline Clear cell & 5 (I5.62\%) & 15.25 & I (3.13\%) & 10.45 & l \\
\hline Other & $2(6.25 \%)$ & 22.72 & $4(12.5 \%)$ & 24.12 & I \\
\hline
\end{tabular}

Notes: *Chemotherapy type includes neoadjuvant chemotherapy (NACT), palliative chemotherapy (PCT) and maintenance chemotherapy (MCT), and histologic type including high grade serous adenocarcinoma, endometrial adenocarcinoma, clear cell carcinoma and unclassified adenocarcinoma.

occurred before or after CA125 turned negative; 5) delayed chemotherapy intervals, longer than 60days nor not and 6) delayed chemotherapy course, more than 2 times nor not (Table 2).

There were four clinical characters affecting CA125 T1/2: the aim of chemotherapy, delayed chemotherapy occurred before or after CA125 turned negative, delayed chemotherapy intervals and delayed chemotherapy course. When delayed chemotherapy took place before CA125 turned negative $(21.47 \pm 7.92 \mathrm{vs} 17.53 \pm 9.25)$ as maintenance chemotherapy or neoadjuvant chemotherapy (23.94 $\pm 6.83 \mathrm{vs} 14.08 \pm 6.88)$ more than 2 times (24.01 $\pm 6.87 \mathrm{vs} 13.47 \pm 6.30$ ), the interval was more than 60 days $(24.04 \pm 6.27 \mathrm{vs} 11.33 \pm 4.18), \mathrm{T} 1 / 2$ increased obviously. There was statistical significance (Table 2).

There were two clinical characters affecting CA125 declined ratio before delayed chemotherapy: delayed chemotherapy occurred before or after CA125 turned negative and delayed intervals. When delayed chemotherapy took place before CA125 turned negative $(0.17 \pm 0.33$ vs 0.49 $\pm 0.55)$ and the interval was more than 60 days $(0.25$ \pm 0.34 vs $0.45 \pm 0.49)$, CA125 declined slower. There was statistical significance (Table 2).

Also, there were four clinical characters affecting CA125 declined ratio after delayed chemotherapy: the aim of chemotherapy, delayed chemotherapy occurred before or after CA125 turned negative, delayed chemotherapy intervals and delayed chemotherapy course.
When delayed chemotherapy took place before CA125 turned negative $(0.04 \pm 0.19 \mathrm{vs} 0.48 \pm 0.34)$ as maintenance chemotherapy or neoadjuvant chemotherapy $(0.20$ $\pm 0.34 \mathrm{vs} 0.49 \pm 0.32)$ more than 2 times $(0.09 \pm 0.23 \mathrm{vs} 0.57$ $\pm 0.31)$, the interval was more than 60 days (0.19 $\pm 0.37 \mathrm{vs} 0.45 \pm 0.32)$, CA125 declined slower. There was statistical significance (Table 2).

As a result: if the delayed chemotherapy took place before CA125 turned negative, and also the interval was more than 60 days, the CA125 half-life and the decreased range of CA125 were totally affected to patients with ovarian epithelial cancer, fallopian tube epithelial cancer and peritoneal epithelial cancer.

\section{Discussion}

How to treat patients with ovarian cancer during coronavirus disease pandemic in 2020? There were few clinical guidelines and expert recommendations during the coronavirus disease pandemic in $2020 .^{2}$ Nonoperative conservative treatment including pharmacological therapies for hormone-sensitive pathologies should be implemented. ${ }^{3}$ Health risk assessment by patient history and coronavirus disease pandemic in 2020 test before elective surgery are pivotal to protect both patients and healthcare providers. ${ }^{4}$ As the epidemic disease was in control, regular treatment such as chemotherapy and operation are orderly recovered and return to normal way. In the special past days, what would happen without 
Table 2 Comparison of CAI25 TI/2 and CAI25 Decrease Extent

\begin{tabular}{|c|c|c|c|}
\hline Sub-Type (N) & $\mathrm{TI} / 2$ & Ratio of DC Before & Ratio of DC After \\
\hline \multicolumn{4}{|c|}{ The aim of chemotherapy: maintain chemotherapy or not } \\
\hline YES(19) & $14.08 \pm 6.88$ & $0.3 I \pm 0.37$ & $0.49 \pm 0.32$ \\
\hline $\mathrm{NO}(13)$ & $23.94 \pm 6.83$ & $0.43 \pm 0.66$ & $0.20 \pm 0.34$ \\
\hline$P$ & 0.002 & 0.084 & 0.027 \\
\hline \multicolumn{4}{|l|}{ FIGO stage } \\
\hline$I-I I(10)$ & $18.53 \pm 5.87$ & $0.36 \pm 0.36$ & $0.43 \pm 0.37$ \\
\hline III-IV(22) & $30.97 \pm 22.04$ & $0.36 \pm 0.54$ & $0.3 I \pm 0.36$ \\
\hline$P$ & 0.13 & 0.99 & 0.453 \\
\hline \multicolumn{4}{|l|}{ Histologic type } \\
\hline High serous(24) & $26.13 \pm 15.77$ & $0.32 \pm 0.52$ & $0.32 \pm 0.35$ \\
\hline Others(8) & $33.03 \pm 30.10$ & $0.49 \pm 0.40$ & $0.38 \pm 0.40$ \\
\hline$P$ & 0.41 & 0.397 & 0.719 \\
\hline \multicolumn{4}{|c|}{ Delayed chemotherapy occurred before or after CAI 25 turned negative } \\
\hline Before (19) & $21.47 \pm 7.92$ & $0.49 \pm 0.55$ & $0.48 \pm 0.34$ \\
\hline After(I3) & $17.53 \pm 9.25$ & $0.17 \pm 0.33$ & $0.04 \pm 0.19$ \\
\hline$P$ & 0.001 & 0.001 & 0.001 \\
\hline \multicolumn{4}{|c|}{ The interval period more than 60 days } \\
\hline $\mathrm{NO}(15)$ & $11.33 \pm 4.18$ & $0.25 \pm 0.34$ & $0.19 \pm 0.37$ \\
\hline YES(I7) & $24.04 \pm 6.27$ & $0.45 \pm 0.49$ & $0.45 \pm 0.32$ \\
\hline $\mathrm{P}$ & 0.000 & 0.013 & 0.033 \\
\hline \multicolumn{4}{|c|}{ Delayed chemotherapy course, more than 2 times nor not. } \\
\hline 2 times & $24.01 \pm 6.87$ & $0.39 \pm 0.61$ & $0.57 \pm 0.31$ \\
\hline More than 3 times & $13.47 \pm 6.30$ & $0.34 \pm 0.39$ & $0.09 \pm 0.23$ \\
\hline$P$ & 0.000 & 0.301 & 0.000 \\
\hline
\end{tabular}

Notes: Chemotherapy type: YES means maintenance chemotherapy (MCT), neoadjuvant chemotherapy and palliative chemotherapy divided to NO. Histologic type including High grade serous adenocarcinoma and others, others including endometrial adenocarcinoma, clear cell carcinoma and unclassified adenocarcinoma.

any treatment for patients with ovarian cancer for more than two months? CI Nagel ${ }^{5}$ had reported that there was no difference detected in survival between patients who required dose adjustments and treatment delays because of side effect. In this report $56 \%$ patients had a delay between 3 to 14 days and $42 \%$ of patients had a delay greater than 14 days. Also, study has reported that ${ }^{6}$ delayed palliative chemotherapy with recurrent ovarian cancer had no adverse effect on survival. As a unique time in 2020, the delayed period was obviously longer than that. More clinical analysis was needed. We choose CA125 changes as indicator to judge the possible sideeffect for overall survival.

\section{CAI 25 Variables}

CA125 is the most common biomarker for diagnosis and follow-up of ovarian cancer. It is a high molecular weight glycoprotein with an elevated serum level $(>35 \mathrm{U} / \mathrm{mL})$ in $50-90 \%$ of patients with ovarian cancer, especially epithelial ovarian cancer, fallopian tube cancer and peritoneal epithelial cancer. Study reported that ${ }^{7}$ the outcomes of patients with pre-treatment serum CA125 $\leq 35 \mathrm{U} / \mathrm{mL}$ were better than those with serum CA125 > $35 \mathrm{U} / \mathrm{mL}$. Many features of CA125 have been explored for an association with overall survival, such as the absolute value of CA125 at different time points during treatment, and pharmacokinetic parameters, including CA125 half-life or time-tonadir. $^{8}$

CA125 changes, including reduction or increase, at a predetermined time point after the beginning of treatment. The best prognostic results appear if CA125 reduced to normal level after the third cycle. ${ }^{9}$ The reduction of CA125 reported a good relationship with longer overall survival, with longer progression free survival and overall survival. ${ }^{10}$ Nadir of CA125 was the most powerful predictor of survival when patients treated with primary debulking surgery or both primary debulking surgery and neoadjuvant chemotherapy-interval debulking surgery. ${ }^{11}$ 
Because we had more neoadjuvant chemotherapy and recurrence cases which did not take operations during delayed intervals, nadir of CA125 were not chosen as treatment effects especially surgery.

Most studies reported that CA125 half-life was most important variable about CA125-related time-to-event. ${ }^{12}$ Since 1988, half-life has been the most studied kinetic parameter in patients with advanced ovarian cancer. Also, we chose CA125 half-life as main interest factor for this study, it is reported that patients with a CA125 half-life less than 20 days had 1.8 times (43 vs 11 months) longer survival times than those with a longer CA125 halflife. ${ }^{13}$ In neoadjuvant chemotherapy and palliative chemotherapy cases after recurrence, the half-life of CA125 was more than 20 days. Although there was no statistical difference, there might be a risk of affecting the survival time. Whether it is affected, further studies will be designed to confirm.

\section{The Explanation of CAI 25 Changes}

Although the CA125 half-life showed no statistical difference, subgroup analysis showed that delayed chemotherapy had a side-effect on the decrease of CA125. CA125 increased in 8 patients after delaying conventional chemotherapy, and 6 patients still showed a small increase in CA125 after the second follow-up chemotherapy. Due to the small increase, it was still within the normal range, and no further treatment was given such as changing the chemotherapy scheme. ${ }^{14}$

In the palliative and neoadjuvant chemotherapy cases, the average level of T1/2 was more than 20 days, especially in patients with high CA125 level before treatment, and then there was no significant difference compared with that in 2019. The possible reasons might be as follows: firstly, due to the small sample size, the increase of standard deviation between samples lead to no statistical difference. Secondly, when the decrease of CA125 was not satisfactory and exceeded the normal range, the drugresistant patients were considered and changed the chemotherapy scheme, which was excluded from the clinical data analysis. As these patients were not included in the statistics, it was possibly the reason for no statistical difference. Therefore, whether it will affect the follow-up progress of the disease, we look forward to the results of survival analysis in the future, which can be preparation for registering clinical follow-up experiments.

\section{Possible of Ovarian Cancer Biomarkers}

Though many patients were diagnosed with evaluated CA125 or HE4 value, there were still some patients who were in low level that they were diagnosed with ovarian cancer. Thus, the present study aimed to explore if there was a role for these parameters that were simple and accessible in the preoperative to find epithelial ovarian cancer, especially in these who were in negative CA125 and HE4 value and make a judgement of the characteristic of the tumor. In recent years, there are more and more advanced equipment to detect pelvic mass, such as different kinds of cancer antigen (CA125, CEA, CA199, AFP), pelvic MRI. ${ }^{15,16}$ When patients are suspicious for developing malignant tumor, more senior technology and consumption are truly needed. Completed blood count and ultrasonography for women are easy to get and at relatively low cost. Chronic inflammation has been associated with the carcinogenesis of different types of tumors, including ovarian cancer, and neutrophil, lymphocyte are common inflammation cells in blood cell. And neutrophilto-lymphocyte ratio and platelet-to-lymphocyte ratio are considered predictive factors for survival in ovarian cancer. ${ }^{17,18}$ More blood biomarkers and image results should be comprehensively considered.

\section{Conclusion}

Although the spread of coronavirus disease pandemic in 2020 continues globally, the subsequent impact on cancer patients during this time remains unclear. Our study found no evidence that the delay in chemotherapy caused by uncontrollable factors during this pandemic affects the decline in CA125 levels and the effects of chemotherapy. However, longer follow-ups to examine the effects on recurrence interval and survival time will be needed in the future.

\section{Highlights}

- Planned chemotherapy for epithelial ovarian, fallopian tube, and peritoneal cancer was delayed by 60 days during coronavirus disease pandemic in 2020.

- Although the half-life of CA125 tended to be higher in those with delayed chemotherapy, the difference was not significant.

- One delay in chemotherapy may not influence the decrease in CA125 levels in epithelial ovarian cancer patients. 


\section{Research Involving Human Participants}

All procedures performed in our studies involving human participants have been approved by the ethics committee of First Affiliated Hospital of Chongqing Medical University and have therefore been performed in accordance with the ethical standards laid down in the 1964 Declaration of Helsinki and its later amendments.

\section{Disclosure}

The authors have declared that no competing interest exists.

\section{References}

1. Frey MK, Ellis AE, Zeligs K, et al. Impact of the coronavirus disease 2019 pandemic on the quality of life for women with ovarian cancer. Am J Obstet Gynecol. 2020;223(5):725.e1-725.e9. doi:10.1016/j. ajog.2020.06.049

2. Rodriguez J, Fletcher A, Heredia F, et al. Alternative management for gynecological cancer care during the COVID-2019 pandemic: a Latin American survey. Int J Gynaecol Obstet. 2020;10:1002. doi:10.1002/ ijgo. 13272

3. Nakayama J, El-Nashar SA, Waggoner S, et al. Adjusting to the new reality: evaluation of early practice pattern adaptations to the COVID-19 pandemic. Gynecol Oncol. 2020;158(2):SS0090. doi:10.1016/j.ygyno.2020.05.028

4. Bogani G, Casarin J, Pinelli C, et al. Management of patients with ovarian cancer in the COVID-19 era. J Surg Oncol. 2020;122 (2):122-123. doi: 10.1002/jso.26057

5. Nagel CI, Backes FJ, Hade EM, et al. Effect of chemotherapy delays and dose reductions on progression free and overall survival in the treatment of epithelial ovarian cancer. Gynecol Oncol. 2012;124 (2):221-224. doi:10.1016/j.ygyno.2011.10.003

6. Pyeon SY, Han GH, Ki KD, et al. Effect of delayed palliative chemotherapy on survival of patients with recurrent ovarian cancer. PLoS One. 2020;15(7):e0236244. doi:10.1371/journal.pone.0236244

7. Yang Z-J, Zhao B-B, Li L. The significance of the change pattern of serum CA125 level for judging prognosis and diagnosing recurrences of epithelial ovarian cancer. J Ovarian Res. 2016;9(1):57. doi:10.1186/ s13048-016-0266-3
8. Zeng J, Huang H, Shan Y, et al. The effect of CA125 nadir level on survival of advanced-stage epithelial ovarian carcinoma after interval debulking surgery. J Cancer. 2011;121(2):3410-3415. doi:10.1016/j. ygyno.2011.01.014

9. Pignata S, Cannella L, Leopardo D, et al. Follow-up with CA125 after primary therapy of advanced ovarian cancer: in favor of continuing to prescribe CA125 during follow-up. Ann Oncol. 2011;22 (Suppl 8):viii40-viii44. doi:10.1093/annonc/mdr470

10. Fleming ND, Cass I, Walsh CS, et al. CA125 surveillance increases optimal resectability at secondary cytoreductive surgery for recurrent epithelial ovarian cancer. Gynecol Oncol. 2011;121(2):249-252. doi:10.1016/j.ygyno.2011.01.014

11. Zeng J, Yin J, Song X, et al. Reduction of CA125 levels during neoadjuvant chemotherapy can predict cytoreduction to no visible residual disease in patients with advanced epithelial ovarian cancer, primary carcinoma of fallopian tube and peritoneal carcinoma. $J$ Cancer. 2016;7(15):2327-2332. doi:10.7150/jca.16761

12. Rustin GJ. Follow-up with CA125 after primary therapy of advanced ovarian cancer has major implications for treatment outcome and trial performances and should not be routinely performed. Ann Oncol. 2011;22(Suppl 8):viii45-viii48. doi:10.1093/annonc/mdr471

13. Colloca G, Venturino A, Governato I. CA125-related tumor cell kinetics variables after chemotherapy in advanced ovarian cancer: a systematic review. Clin Transl Oncol. 2016;18(8):813-824. doi:10.1007/s12094-015-1441-5

14. Chiang AJ, Chen J, Chung Y-C, et al. A longitudinal analysis with CA-125 to predict overall survival in patients with ovarian cancer. J Gynecol Oncol. 2014;25(1):51-57. doi:10.3802/jgo.2014.25.1.51

15. Felder M, Kapur A, Gonzalez-Bosquet J, et al. MUC16 (CA125): tumor biomarker to cancer therapy, a work in progress. Mol Cancer. 2014;13(1):129. doi:10.1186/1476-4598-13-129

16. Dochez V, Caillon H, Vaucel E, et al. Biomarkers and algorithms for diagnosis of ovarian cancer: CA125, HE4, RMI and ROMA, a review. J Ovarian Res. 2019;12(1):28. doi:10.1186/s13048-0190503-7

17. Zhao Z, Zhao X, Lu J, et al. Prognostic roles of neutrophil to lymphocyte ratio and platelet to lymphocyte ratio in ovarian cancer: a meta-analysis of retrospective studies. Arch Gynecol Obstet. 2018;297(4):849-857. doi:10.1007/s00404-018-4678-8

18. Saed GM, Diamond MP, Fletcher NM. Updates of the role of oxidative stress in the pathogenesis of ovarian cancer. Gynecol Oncol. 2017;145(3):595-602. doi:10.1016/j.ygyno.2017.02.033

\section{Publish your work in this journal}

Cancer Management and Research is an international, peer-reviewed open access journal focusing on cancer research and the optimal use of preventative and integrated treatment interventions to achieve improved outcomes, enhanced survival and quality of life for the cancer patient.
The manuscript management system is completely online and includes a very quick and fair peer-review system, which is all easy to use. Visit http://www.dovepress.com/testimonials.php to read real quotes from published authors. 